\title{
APPROXIMATELY TRANSITIVE DIFFEOMORPHISMS OF THE CIRCLE
}

\author{
J. HAWKINS ${ }^{1}$ AND E. J. WOODS
}

\begin{abstract}
We prove that every $C^{3}$ diffeomorphism of the circle with an irrational rotation number (and some $C^{2}$ diffeomorphisms as well) are approximately transitive. This provides a class of examples of approximately transitive transformations which are smooth but non-measure-preserving.
\end{abstract}

1. Introduction. This paper provides a class of examples of $C^{\infty}$ approximately transitive integer actions. The concept of approximate transitivity was introduced by Connes and Woods to provide necessary and sufficient conditions for a hyperfinite von Neumann factor to be ITPFI [1]. Examples of both approximately transitive (AT) and non-AT group actions have been given in [1]. In particular, it is easy to obtain both AT and non-AT diffeomorphisms of $T^{2}=\mathbf{R}^{2} / \mathbf{Z}^{2}$. (For AT examples take rotation in the $x$ direction by $\alpha$, rotation in the $y$ direction by $\beta$, with $\alpha$ and $\beta$ two rationally independent numbers in $(0,1)$. For non-AT examples, take the ergodic automorphisms induced by $U_{n}=\left(\begin{array}{cc}n+1 & 1 \\ n & 1\end{array}\right)$ on $\mathbf{R}^{2} / \mathbf{Z}^{2}$.)

It has been shown in [3] and [4], to mention only two, that diffeomorphisms of the circle exhibit many different types of measure theoretic behavior. For example, Katznelson [4] proved that any $C^{2}$ diffeomorphism of the circle with irrational rotation number not of constant type (i.e., having unbounded continued fraction coefficients) is orbit equivalent to an odometer of product type, hence is ITPFI.

Using methods similar to those employed in [4] we show that every $C^{2}$ diffeomorphism of $T^{1}$ whose rotation number is not of constant type and every $C^{3}$ diffeomorphism with irrational rotation number is approximately transitive. Examples of type III diffeomorphisms of manifolds whose associated ergodic flows are built under constant ceiling functions with base transformations $C^{3}$ diffeomorphisms of $T^{1}$ are given in [2]; these diffeomorphisms are therefore ITPFI.

The first section of this paper defines a property which will be shown to imply approximate transitivity; we call it Property F. It is one possible generalization of Funny Rank One (a property defined in the measure-preserving case [1]) to the nonsingular case. In the second section it is shown that using some estimates from Herman's thesis [3], all $C^{2}$ diffeomorphisms of the circle with irrational rotation

Received by the editors January 10, 1983 and, in revised form, June 27, 1983.

1980 Mathematics Subject Classification. Primary 46L35, 58F11, 47A35.

Key words and phrases. Classification of von Neumann factors, orbit equivalence of ergodic transformations, diffeomorphisms of the circle.

'Supported in part by National Science Foundation Grant MCS-8102399.

C1984 American Mathematical Society $0002-9939 / 84 \$ 1.00+\$ .25$ per page 
number having unbounded coefficients satisfy Property F. In the $C^{3}$ case, we apply Herman's Theorem to obtain approximate transitivity for all irrational rotation numbers.

2. Property F implies AT. We begin with the definition of approximate transitivity for the case of a nonsingular integer action (cf. [1] for the most general definition).

Let $(X, \Re, \mu)$ denote a Lebesgue space and $T:(X, \Re, \mu) \hookleftarrow$ a nonsingular, invertible transformation of $(X, \mathscr{B}, \mu)$.

Definition 2.1. The action of $T$ on $(X, \mathscr{B}, \mu)$ is approximately transitive $(A T)$ if given $\varepsilon>0$ and $\varphi_{1}, \ldots, \varphi_{n} \in L_{+}^{1}(X, \mu)$, there exist $\varphi \in L_{+}^{1}(X, \mu)$ and $\lambda_{1}, \ldots, \lambda_{n} \in$ $l_{+}^{1}(Z)$ such that

$$
\left\|\varphi_{j}-\sum_{k \in \mathbf{Z}} \lambda_{j k} \cdot \varphi \circ T^{-k} \frac{d T_{*}^{-k} \mu}{d \mu}\right\|_{1}<\varepsilon
$$

for $j=1, \ldots, n$.

Here, $d T_{*}^{-k} \mu / d \mu$ denotes the Radon-Nikodym derivative of $T_{*}^{-k} \mu$ with respect to $\mu$.

It is easy to see that an AT transformation is ergodic. Connes and Woods also proved that a necessary and sufficient condition for a hyperfinite von Neumann factor to be ITPFI is that the associated flow of weights be AT [1].

We now introduce the concept of Property $F$ and prove that it provides a sufficient condition for approximate transitivity.

Definition 2.2. A nonsingular transformation $T$ of $(X, \Re, \mu)$ has Property $\mathrm{F}$ if given any measurable sets $A_{1}, \ldots, A_{m} \subseteq X$ and $\delta>0$ there exist a set $B \subseteq X$, a sequence $n_{1}, \ldots, n_{s}$ of distinct integers, subsequences $n_{j 1}, \ldots, n_{j k_{j}}$ for each $j$, and sets of real numbers $\left\{\gamma_{j i}\right\}_{i=1}^{k_{j}}, \gamma_{j i} \neq 0$ for each $j=1, \ldots, m$, satisfying

(i) $T^{n_{1}} B, \ldots, T^{n_{s}} B$ are all disjoint;

(ii) $\mu\left(A_{j} \Delta \cup_{i=1}^{k_{j}} T^{n_{j i} B}\right)<\delta$; and

(iii) for each $n_{j i} \leqslant n_{s}$, there exists $\gamma_{j i} \neq 0$ such that

$$
\left|\frac{d T_{*}^{n_{j i} \mu}}{d \mu}(x) \cdot \gamma_{j i}^{-1}-1\right|<\delta \text { for } \mu \text {-a.e. } x \in B \text {. }
$$

To show that Property $F$ implies approximate transitivity, we first show that equation (1) of Definition 2.1 is satisfied if we consider simple functions $\varphi_{1}, \ldots, \varphi_{n}$. In particular, $\varphi$ will be the characteristic function of $B$ in Definition 2.2, and a routine calculation shows that $\lambda_{j k}$ will depend on the appropriate $\gamma_{j i}$ or will be equal to zero. To show (2.1) is satisfied for arbitrary functions $\varphi_{1}, \ldots, \varphi_{n} \in L_{+}^{1}(X, \mu)$, we just approximate by simple functions. We have outlined the proof of the following.

Proposition 2.3. If $T:(X, \mu) \hookleftarrow$ has Property $\mathrm{F}$, then it is $A T$.

REMARKS. 1. It is easy to see that odometers of product type have Property F (see [4] for definition) and, therefore, are AT.

2. In the measure-preserving case, it is known that Rank One and Funny Rank One imply that a transformation is AT [1]. Property F is one generalization of Funny Rank One to the non-measure-preserving, but nonsingular, case. 
3. Diffeomorphisms of the circle. Let $f$ denote an orientation preserving diffeomorphism of $T^{1}=\mathbf{R} / \mathbf{Z}$. We write $\alpha=\rho(f)$ for the rotation number of $f$; we assume that $\alpha$ is irrational and write $\left.\alpha=1\lrcorner a_{1}+1\right\lrcorner a_{2}+\cdots=\left[a_{1}, a_{2}, \ldots\right)$ for the continued fraction expansion of $\alpha$. If $\sup _{n} a_{n}<\infty$, we say $\alpha$ is of constant type. The $n$th convergent of $\alpha$ is denoted by $p_{n} / q_{n}$, where $p_{n}$ and $q_{n}$ satisfy the recursion formulae: $p_{0}=0, p_{1}=q_{0}=1, p_{n}=a_{n} p_{n-1}+p_{n-2}, q_{n}=a_{n} q_{n-1}+q_{n-2}$, for every $n \geqslant 2$.

Let $Q_{n}(f)$ denote the partition of $T^{1}$ given by the points $\left\{f^{j}\left(x_{0}\right)\right\}_{j=0}^{q_{n}+q_{n+1}-1}$. If $n$ is even the partition $Q_{n}(f)$ consists of a disjoint union of intervals $\left\{f^{j}\left[x_{0}, f^{q_{n}} x_{0}\right)\right\}_{j=0}^{q_{n+1}-1}$ and $\left\{f^{k}\left[f^{q_{n+1}} x_{0}, x_{0}\right)\right\}_{k=0}^{q_{n}-1}$ which cover $T^{1}$. If we let

$$
E_{n}=\bigcup_{j=0}^{q_{n+1}-1} f^{j}\left[x_{0}, f^{q_{n}} x_{0}\right) \text { and } F_{n}=\bigcup_{k=0}^{q_{n}-1} f^{k}\left[f^{q_{n+1}} x_{0}, x_{0}\right),
$$

then we need some lemmas to estimate their relative sizes. In particular, to prove $f$ has Property $\mathrm{F}$ we want to approximate measurable sets by intervals in $E_{n}$ so we need to insure that the measure of $F_{n}$ is small. This is true only when $\alpha$ is not of constant type.

The following lemmas have now become standard estimates used in proving results on diffeomorphisms of the circle. For this reason, we refer the reader to [3] for their proofs. We denote by $D^{2}\left(T^{1}\right)$ the group of all $C^{2}$ orientation preserving diffeomorphisms of the circle. We will denote Lebesgue measure on $T^{1}$ by $m$.

Lemma 3.1 (DenJoy's INEQUALITy [3, V1.4.4, p. 75]). Let $f \in D^{2}\left(T^{1}\right), \rho(f)=\alpha$ $\in \mathbf{R} \backslash \mathbf{Q}, p_{n} / q_{n}$ a continued fraction convergent of $\alpha$. Then

$$
e^{-V} \leqslant D f^{ \pm q_{n}} \leqslant e^{V} \text { with } V=\operatorname{Var}(\log D f) .
$$

Lemma $3.2\left[3, \mathrm{~V} 1.6 .2\right.$, p. 77]. Let $f \in D^{2}\left(T^{1}\right), \rho(f)=\alpha \in \mathbf{R} \backslash \mathbf{Q}, p_{n} / q_{n}$ a convergent of $\alpha$. Assume $y, z \in\left[x_{0}, f^{q_{n}} x_{0}\right]$. Then for all $0 \leqslant j<q_{n+1}$,

$$
e^{-V} \leqslant D f^{j}(y) / D f^{j}(z) \leqslant e^{V}, \text { with } V=\operatorname{Var}(\log D f) .
$$

We also use the following result.

Lemma 3.3 (Denjoy's Theorem [3, VI.5.5, p. 76]). Let $f \in D^{2}\left(T^{1}\right), \rho(f)=\alpha \in$ $\mathbf{R} \backslash \mathbf{Q}$. Then

$$
f=h^{-1} \circ R_{\alpha} \circ h
$$

with h a homeomorphism of $T^{1}$.

Given $f \in D^{2}\left(T^{1}\right)$ with $\alpha \in \rho(f) \in \mathbf{R} \backslash \mathbf{Q}$, we define

$$
g_{n}=\frac{1}{n} \sum_{i=0}^{n-1}\left(f^{i}-i \alpha\right) \in D^{2}\left(T^{1}\right) \text {. }
$$

We now let $f_{n}=g_{n} \circ f \circ g_{n}^{-1}$. Then the following lemma holds.

LEMMA 3.4 [3, VII.2.5.1]. With notation as above, $V_{n}=\operatorname{Var}\left(\log D f_{n}\right) \rightarrow 0$ as $n \rightarrow \infty$.

We use those lemmas to prove the following estimate, which is crucial for our main result. 
Lemma 3.5 [3, VII.2.5.3, p. 95]. Let $f \in D^{2}\left(T^{1}\right)$ with $\rho(f)=\alpha \in \mathbf{R} \backslash \mathbf{Q}$. If

$$
C_{n}=\sup _{0 \leqslant i<q_{n+1}}\left(\operatorname{Max}_{x \in T^{1}}\left(\operatorname{Max}_{y, z \in\left[x, f^{u_{n} x}\right]} \frac{D f^{i}(y)}{D f^{i}(z)}\right)\right),
$$

then $C_{n} \rightarrow 1$ as $n \rightarrow \infty$.

Proof. We write $f=g_{n}^{-1} \circ f_{n} \circ g_{n}$, with $g_{n}=n^{-1} \sum_{i=0}^{n-1}\left(f^{i}-i \alpha\right)$ and $f_{n}=$ $g_{n} \circ f \circ g_{n}^{-1}$. Given $\varepsilon>0$, we choose an $N$ large enough so that for all $n \geqslant N$, $\operatorname{Var}\left(\log D f_{n}\right)<\varepsilon$ (by Lemma 3.4). Recall that for any point $x \in T^{1}$, the intervals given by $\left\{f^{j}\left[x, f^{q_{n}} x\right)\right\}, 0 \leqslant j<q_{n+1}$, and $\left\{f^{k}\left[f^{q_{n+1}} x, x\right)\right\}, 0 \leqslant k<q_{n}$, cover $T^{1}$ and are pairwise disjoint if $n$ is even and $q_{n}$ is the denominator of a convergent of $\alpha$. Furthermore, by Lemma 3.3 as $n \rightarrow \infty$, the lengths of the intervals shrink to 0 . We choose $N_{1} \geqslant N$ so large that for all $n \geqslant N_{1}$ we have

$$
\max D g_{N}^{-1}(y) / \min D g_{N}^{-1}(y)<1+\varepsilon,
$$

where the maximum and minimum are taken over all $y$ in any one interval of $\cup_{\substack{q_{n+1} \\ q_{n=0}}} f^{j}\left(\left[x, f^{q_{n}} x\right]\right)$. We write

$$
\frac{D f^{i}(y)}{D f^{i}(z)}=\frac{D g_{N}^{-1}\left(f_{N}^{i} \circ g_{N} y\right) \cdot D f_{N}^{i}\left(g_{N} y\right) \cdot D g_{N}(y)}{D g_{N}^{-1}\left(f_{N}^{i} \circ g_{N} z\right) \cdot D f_{N}^{i}\left(g_{N} z\right) \cdot D g_{N}(z)} \text {. }
$$

Since $f_{N}^{i}\left(g_{N} y\right)\left(=g_{N}\left(f^{i} y\right)\right)$ and $f_{N}^{i}\left(g_{N} z\right)$ are in the same subinterval of $Q_{n}(f)$ if $y$ and $z$ are for suitable $x_{0} \in T^{1}$ and for $0 \leqslant i<q_{n+1}$, then using Lemma 3.2 and the above estimates we have

$$
D f^{i}(y) / D f^{i}(z) \leqslant(1+\varepsilon)^{2} e^{\varepsilon}
$$

for all $1 \leqslant i<q_{n+1}$ and all $n \geqslant N_{1}$. Then as $N_{1} \rightarrow \infty$ it follows that

$$
\sup _{0 \leqslant i<q_{N_{1}}+1}\left(\operatorname{Max}_{x \in T^{i}}\left(\operatorname{Max}_{y, z \in\left[x, f^{\left.q_{N_{1}} x\right]}\right.} \frac{D f^{i}(y)}{D f^{i}(z)}\right)\right) \rightarrow 1
$$

as claimed.

From 3.1 and 3.4 we also have the following estimate from [4] on the size of $F_{n}$.

LEMMA 3.6. If $f \in D^{2}\left(T^{1}\right)$ and $\rho(f)=\alpha$ is irrational, then for any $\varepsilon>0$ there exists $N \in \mathbf{N}$ such that for all $n \geqslant N, m\left(F_{n}\right)<\varepsilon /\left(1-(1-\varepsilon)^{q_{n+2} \cdot q_{n}^{-1}}\right)$. (Therefore, if $\alpha$ has unbounded coefficients, $q_{n_{k}+2} q_{n_{k}}^{-1} \rightarrow \infty$ for a subsequence of integers, so $m\left(F_{n_{k}}\right)$ $\rightarrow 0$.)

We are now ready to prove that diffeomorphisms of the circle are approximately transitive under certain conditions.

THEOREM 3.7. If $f \in D^{2}\left(T^{1}\right)$ and $\rho(f)$ is not of constant type, then $f$ has Property F.

Proof. Suppose we are given some measurable sets $A_{1}, \ldots, A_{m} \subseteq T^{1}$ and a $\delta>0$. We choose a point of $A_{1}$; call it $x_{0}$. We will approximate $A_{1}, \ldots, A_{m}$ by intervals in the partition $Q_{n}(f)$ as defined earlier. We first choose $n$ even and large enough so that intervals in $Q_{n}(f)$ cover $A_{1}, \ldots, A_{m}$ to within $\delta / 2$ in measure. We define 
$B=\left[x_{0}, f^{q_{n}} x_{0}\right)$, and choose $n$ larger if necessary (and even) so that

$$
m\left(T^{1} \backslash \bigcup_{k=0}^{q_{n+1}-1} f^{k} B\right)<\delta / 2 \quad \text { (using Lemma 3.6). }
$$

Applying Lemma 3.5 we choose $n$ still larger and even so that $\left|C_{n}-1\right|<\delta / 4$ where

$$
C_{n}=\sup _{0 \leqslant i<q_{n+1}}\left(\operatorname{Max}_{x \in T^{i}}\left(\operatorname{Max}_{y, z \in\left[x, f^{\left.q_{n} x\right]}\right.} \frac{D f^{i}(y)}{D f^{i}(z)}\right)\right) .
$$

We can easily verify that the conditions of Property $F$ are satisfied. We choose our sequence $n_{1}, \ldots, n_{s} \leqslant q_{n+1}-1$, so we have $f^{n_{1}} B, f^{n_{2}} B, \ldots, f^{n_{s}} B$ all disjoint and (i) holds. Then for each $j$, we choose $n_{j i} \leqslant n_{s}$ to be the appropriate powers of $f$ which carry $B$ onto intervals of $E_{n} \subseteq Q_{n}(f)$ which approximate $A_{j}$. Finally by Lemma 3.5 and our choice of $n$, we can choose $\gamma_{j i}=\sup _{x \in B} D f^{n_{j i}}(x)$. Then (iii) will follow.

Corollary 3.8. If $f \in D^{2}\left(T^{1}\right)$ and $\rho(f)$ is not of constant type, then $f$ is $A T$.

COROllary 3.9. If $f \in D^{3}\left(T^{1}\right)$ and $\rho(f)$ is irrational, then $f$ is $A T$.

Proof. Suppose $\alpha=\rho(f)$ has unbounded coefficients. We then apply 3.8. If $\alpha=\rho(f)$ is of constant type then we have that $f$ is $C^{1}$ conjugate to $R_{\alpha}$ by the main theorem of Herman's thesis [3]. Hence $f$ is measure theoretically isomorphic to $R_{\alpha}$. Since ergodic rotations of compact abelian groups are AT, and approximate transitivity is an isomorphism invariant [1], then it follows that $f$ is AT.

\section{REFERENCES}

1. A. Connes and E. J. Woods, Approximately transitive flows and ITPFI factors (to appear).

2. J. Hawkins, Non-ITPFI diffeomorphisms, Israel J. Math. 42 (1982), 117-131.

3. M. R. Herman, Sur la conjugaison différentiable des difféomorphisms du cercle à des rotations, Inst. Hautes Études Sci. Publ. Math. 49 (1979), 5-234.

4. Y. Katznelson, The action of diffeomorphisms of the circle on Lebesgue measure, J. Analyse Math. 36 (1979), 156-166.

5. E. J. Woods, ITPFI factors-a survey, Queen's Univ., preprint, 1980.

Department of Mathematics, State University of New York, Stony Brook, New York 11794

Department of Mathematics, Queen's University, Kingston, Ontario, Canada K7L 3N6 